\title{
Examination of the Mixed Layer Deepening Process during Convection Using LES
}

\author{
YIGN NOH AND GAHYUN GOH \\ Department of Atmospheric Sciences/Global Environmental Laboratory, Yonsei University, Seoul, South Korea \\ SIEGFRIED RAASCH \\ Institute of Meteorology and Climatology, University of Hannover, Hannover, Germany
}

(Manuscript received 5 May 2009, in final form 9 February 2010)

\begin{abstract}
Analysis of large-eddy simulation data of the ocean mixed layer under convection reveals that the contribution from wind stress decreases with time as a result of inertial oscillation in the extratropical ocean and that it leads to a rapid increase of the bulk and gradient Richardson number at the mixed layer depth. The criteria for the mixed layer deepening in the widely used mixed layer models, such as the Niiler-Kraus (NK) model, the Price-Weller-Pinkel (PWP) model, and the $K$-profile parameterization (KPP) model, are examined in view of these results, and its implication on the model predictability is discussed.
\end{abstract}

\section{Introduction}

The bulk models of the ocean mixed layer usually calculate the growth of mixed layer depth (MLD) based on a certain criterion at the MLD. In particular, this approach is effective in dealing with the mixed layer deepening under convection, in which the mixed layer remains well mixed.

For example, Niiler and Kraus (1977, hereafter NK) suggested that the entrainment velocity of the mixed layer deepening $w_{e}(=d h / d t)$ can be predicted from the formula for the buoyancy flux at the $\operatorname{MLD}(z=-h)$, $-\overline{b w}_{h}\left(=w_{e} \Delta B\right)$, such as

$$
-\overline{b w}_{h}=n Q_{0}+2 m_{0} u_{*}^{3} / h+m_{s} w_{e}(\Delta \mathbf{U})^{2} / h,
$$

where $u_{*}$ is the frictional velocity, $Q_{0}$ is the negative surface buoyancy flux, $B(\mathbf{U})$ is the mean buoyancy (velocity) in the mixed layer, and $\Delta B(\Delta \mathbf{U})$ is its jump at $z=-h$. Davis et al. (1981b) suggested optimum values for the empirical constants as $n=0.21, m_{0}=0.39$, and $m_{s}=0.48$. The last term, which represents the contribution from velocity shear at the MLD, is often neglected (Gaspar 1988).

Corresponding author address: Yign Noh, Department of Atmospheric Sciences/Global Environmental Laboratory, Yonsei University, 134 Shinchon-dong, Seodaemun-gu, Seoul 120-749, South Korea.

E-mail: noh@yonsei.ac.kr
Alternatively, Price et al. (1986, hereafter PWP) suggested that the deepening of the MLD is determined by the condition that the gradient Richardson number defined by

$$
\mathrm{Ri}_{g}=\frac{\partial B / \partial z}{|\partial \mathbf{U} / \partial z|^{2}}
$$

and the bulk Richardson number defined by

$$
\mathrm{Ri}_{b}=\frac{h \Delta B}{|\Delta \mathbf{U}|^{2}}
$$

remain constant at the MLD. A modified form of $\mathrm{Ri}_{b}$ is used by Large et al. (1994) in the $K$-profile parameterization (KPP) model such as

$$
\mathrm{Ri}_{b}^{*}=\frac{h \Delta B}{|\Delta \mathbf{U}|^{2}+V_{t}^{2}},
$$

where $V_{t}^{2}$ represents the contribution from the unresolved shear that becomes important when the mean velocity disappears.

Meanwhile, it has been well known that the resonant wind forcing (i.e., the wind that rotates at the inertial frequency) induces much stronger mixed layer deepening by building up momentum within the mixed layer, compared to the off-resonant wind forcing (Skyllingstad et al. 2000; Large and Crawford 1995; Crawford and Large 1996). The resonant wind forcing is equivalent to the 

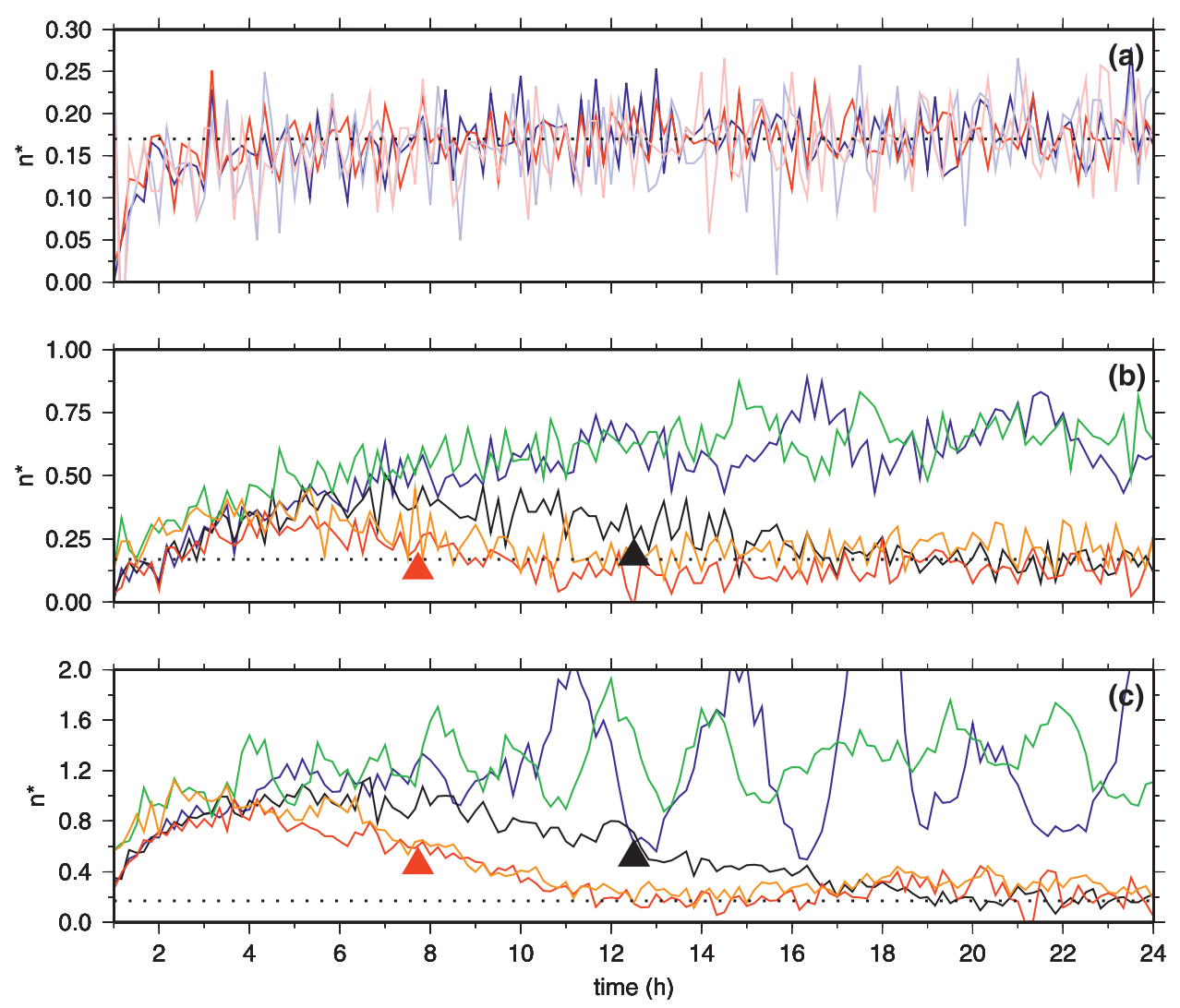

FIG. 1. Time series of $n^{*}$ [blue (green): $\phi=0^{\circ} \mathrm{N}$; black (no): $\phi=30^{\circ} \mathrm{N}$; and red (orange): $\phi=55^{\circ} \mathrm{N}$ for without (with) LC]. The time corresponding to $\pi / f$ is marked by an arrow for each experiment. Dotted horizontal lines represent $n^{*}=0.17$ : (a) $u^{*}=0 \mathrm{~m} \mathrm{~s}^{-1}$ (light color: $Q_{0}=2.5 \times 10^{-7} \mathrm{~m}^{2} \mathrm{~s}^{-3}$; dark color: $\left.Q_{0}=5 \times 10^{-7} \mathrm{~m}^{2} \mathrm{~s}^{-3}\right)$, (b) $u^{*}=0.01 \mathrm{~m} \mathrm{~s}^{-1}$ and $Q_{0}=2.5 \times 10^{-7} \mathrm{~m}^{2} \mathrm{~s}^{-3}$, and (c) $u^{*}=0.02 \mathrm{~m} \mathrm{~s}^{-1}$ and $Q_{0}=$ $5 \times 10^{-7} \mathrm{~m}^{2} \mathrm{~s}^{-3}$.

constant wind forcing in the equatorial ocean, so one can expect that the effect of wind forcing is much stronger for the mixed layer deepening in the equatorial ocean than in the high-latitude ocean, when a constant wind stress is applied. It is not clear, however, whether these effects are properly represented in the criteria (1)-(4).

Recent progress of large-eddy simulation (LES) of the ocean mixed layer (e.g., Sullivan et al. 2007; Noh et al. 2004; Skyllingstad et al. 2000) allows us to examine the hypotheses used in ocean mixed layer models. Therefore, in the present paper we examined the criteria in widely used models (1)-(4) by analyzing the LES data. Especially we focused on the role of wind forcing during the deepening of the convective mixed layer and its dependence on latitude.

\section{Simulation}

The LES model used in the present simulation is the same as used in Noh et al. (2004; 2009) in which Langmuir circulation (LC) is realized by the Craik-Leibovich vortex force (Craik and Leibovich 1976) and wave breaking is represented by stochastic forcing. The model domain is $300 \mathrm{~m}$ in both horizontal directions and $80 \mathrm{~m}$ in the vertical direction with a uniform grid size of $1.25 \mathrm{~m}$ along all directions. A free-slip boundary condition is applied at the bottom.

Integration was initiated by applying a negative surface buoyancy flux and wind stress to a motionless fluid. The initial density was uniform down to $z=10 \mathrm{~m}$ and was linearly stratified below with $N^{2}=10^{-4} \mathrm{~s}^{-2}$. A series of experiments was performed with various combinations of $u_{*}=0,0.01$, and $0.02 \mathrm{~m} \mathrm{~s}^{-1}$ and $Q_{0}=1.25$, 2.5 , and $5 \times 10^{-7} \mathrm{~m}^{2} \mathrm{~s}^{-3}$, covering a range of heat flux $100-1000 \mathrm{~W} \mathrm{~m}^{-2}$ and latitudes $\phi=0^{\circ}, 30^{\circ}$, and $55^{\circ} \mathrm{N}$. The conditions for present simulations are similar to those in the one-dimensional mixed layer model experiments (Martin 1985; Large et al. 1994; D'Alessio et al. 1998; Noh and Kim 1999). Both cases with and without LC were simulated to examine its effect.

In the analysis of LES data, $h$ is calculated by the depth of the minimum $\overline{b w}$. Also, $\Delta B$ and $\Delta \mathbf{U}$ are calculated by 



FIG. 2. Evolution of vertical profiles of $S^{2}$ with time ( $\Delta t=2 \mathrm{~h} ; u^{*}=0.02 \mathrm{~m} \mathrm{~s}^{-1} ; Q_{0}=5 \times$ $10^{-7} \mathrm{~m}^{2} \mathrm{~s}^{-3}$; without LC): (a) $\phi=0^{\circ} \mathrm{N}$ and (b) $\phi=55^{\circ} \mathrm{N}$.

the difference between the value averaged over the mixed layer and that at the MLD where $\overline{b w}$ disappears.

\section{Results}

\section{a. Examination of the criteria at the $M L D$}

To estimate the efficiency of entrainment, we calculated the time series of $n^{*}\left(=-\overline{b w}_{h} / Q_{0} ;\right.$ Fig. 1). Here, $n^{*}$ is calculated from the variation of potential energy, following Ayotte et al. (1996). In free convection, in which $n^{*}$ is equivalent to $n$, it approaches a constant value $n \cong$ 0.17 after the initial stage, regardless of $Q_{0}$ and the Coriolis parameter $f$ (Fig. 1a). The value of $n$ is consistent with that suggested for the convective boundary layer $(n \sim 0.1-0.3$; Stull 1986), although it is slightly smaller than the value $n=0.21$ used in the NK model. Here, $n^{*}$ reaches a constant value after about $10 h / w^{*}\left(\sim 7 \times 10^{3} \mathrm{~s}\right)$ with $w^{*}=\left(Q_{0} h\right)^{1 / 3}$, which corresponds to the time scale for the convective boundary layer to reach quasi-equilibrium state (Moeng and Sullivan 1994).

It has been suggested that free convection is suppressed by the Coriolis force, if Ro $\left\{=\left[Q_{0} /\left(f^{3} h^{2}\right)\right]^{1 / 2}\right\}$ becomes smaller than one (Denbo and Skyllingstad 1996; Raasch and Etling 1991; Wang 2003). Its effect is negligible, however, in shallow convection as in the present case where Ro is much larger than one.

In the presence of wind stress, however, the evolution of $n^{*}$ follows very different patterns depending on latitude (Figs. 1b,c). When $\phi=0^{\circ}$, the value of $n^{*}$ continues to increase, until it reaches a critical level. Thereafter, $n^{*}$ ceases to increase and instead shows an oscillating pattern. This oscillation is due to the generation of intermittent 

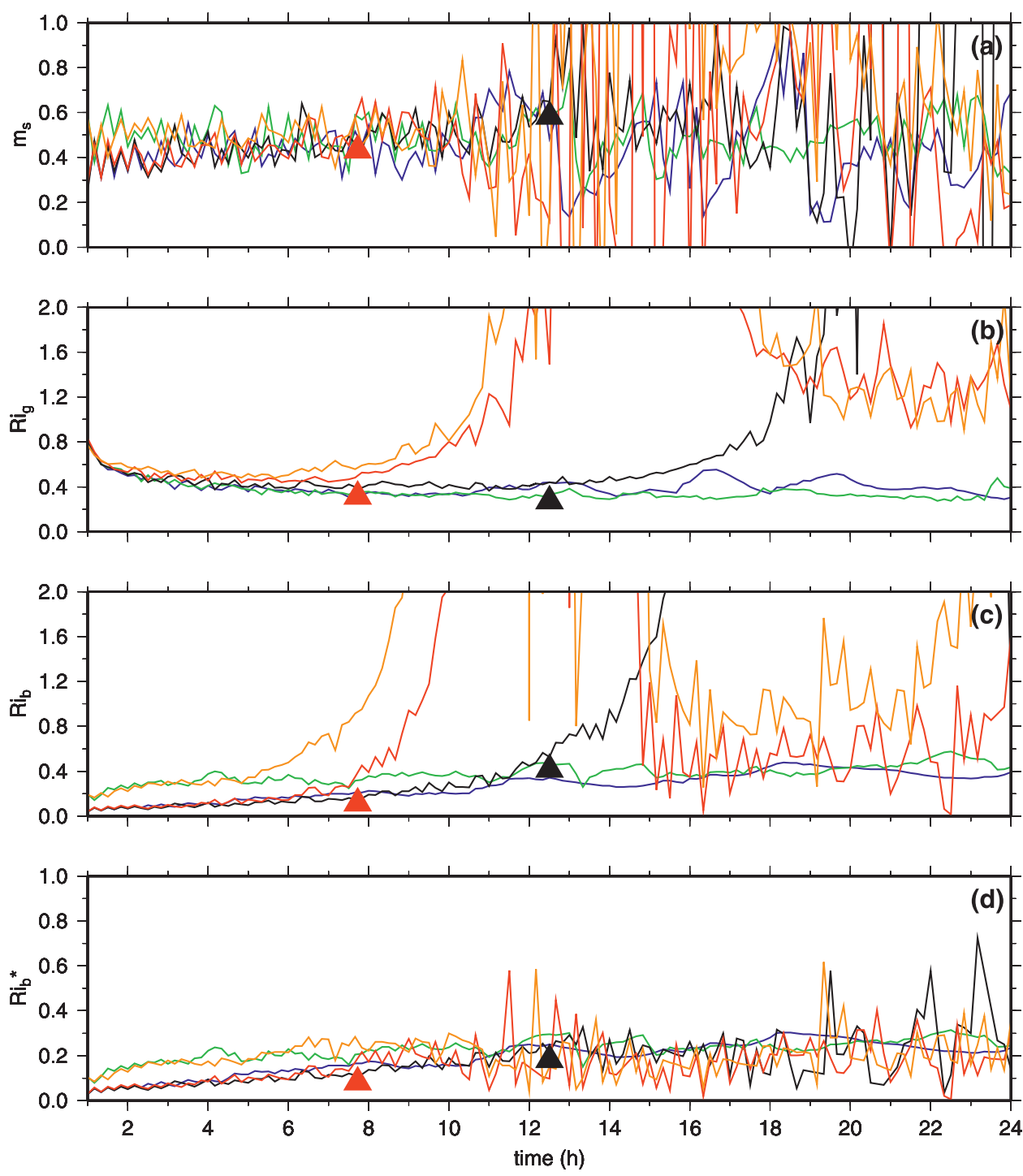

FIG. 3. Time series of Richardson numbers and $m_{s}$ at the MLD $\left[u^{*}=0.02 \mathrm{~m} \mathrm{~s}^{-1}\right.$ and $Q_{0}=5 \times$ $10^{-7} \mathrm{~m}^{2} \mathrm{~s}^{-3}$; blue (green): $\phi=0^{\circ} \mathrm{N}$; black (no): $\phi=30^{\circ} \mathrm{N}$; red (orange): $\phi=55^{\circ} \mathrm{N}$ for without (with) LC]. The time corresponding to $\pi / f$ is marked by an arrow for each experiment: (a) $m_{s}$, (b) $\mathrm{Ri}_{g}$, (c) $\mathrm{Ri}_{b}$, and (d) $\mathrm{Ri}_{b}^{*}$.

turbulence at the MLD by the buildup of shear beyond a critical level, as is often observed in the stable boundary layer (e.g., Mahrt 1999; Doran 2004).

On the other hand, when $\phi=30^{\circ}$ or $55^{\circ} \mathrm{N}$, although it increases initially in a similar fashion to the case of $\phi=$ $0^{\circ}, n^{*}$ starts to decrease after $t \sim \pi / 2 f$ and ultimately approaches a value indistinguishable from that in free convection. It implies that the contribution from wind forcing to the mixed layer deepening vanishes when $t \gg$ $\pi / f$, as a result of inertial oscillation. The situation is similar to the LES results by Skyllingstad et al. (2000), in which off-resonant wind stress produces much slower mixed layer deepening than resonant wind stress.
Figure 1 also shows that the presence of LC causes no noticeable difference. It implies that the vertical mixing is dominated by convective eddies in the present simulation, even though the distinctive pattern of LC is found in the velocity field, as expected from the simulation conditions $\mathrm{La}_{t}\left[=\left(u_{*} / U_{s}\right)^{1 / 2}\right]=0.45$ and $\mathrm{Ho}\left[=2 Q_{0} /\right.$ $\left.\left(k U_{s} u_{*}^{2}\right)\right]=0.26$ (Fig. 1b) or $\mathrm{La}_{t}=0.64$ and $\mathrm{Ho}=0.13$ (Fig. 1c), where $U_{s}$ is the Stokes velocity at the surface and $k$ is the wavenumber ( $\mathrm{Li}$ et al. 2005).

The evolution of vertical profiles of velocity shear $S^{2}=|\partial \mathbf{U} / \partial z|^{2}$ clearly identifies the effect of inertial oscillation (Fig. 2). Here, the depth of the maximum $S^{2}$ coincides with the MLD because of strong convective 

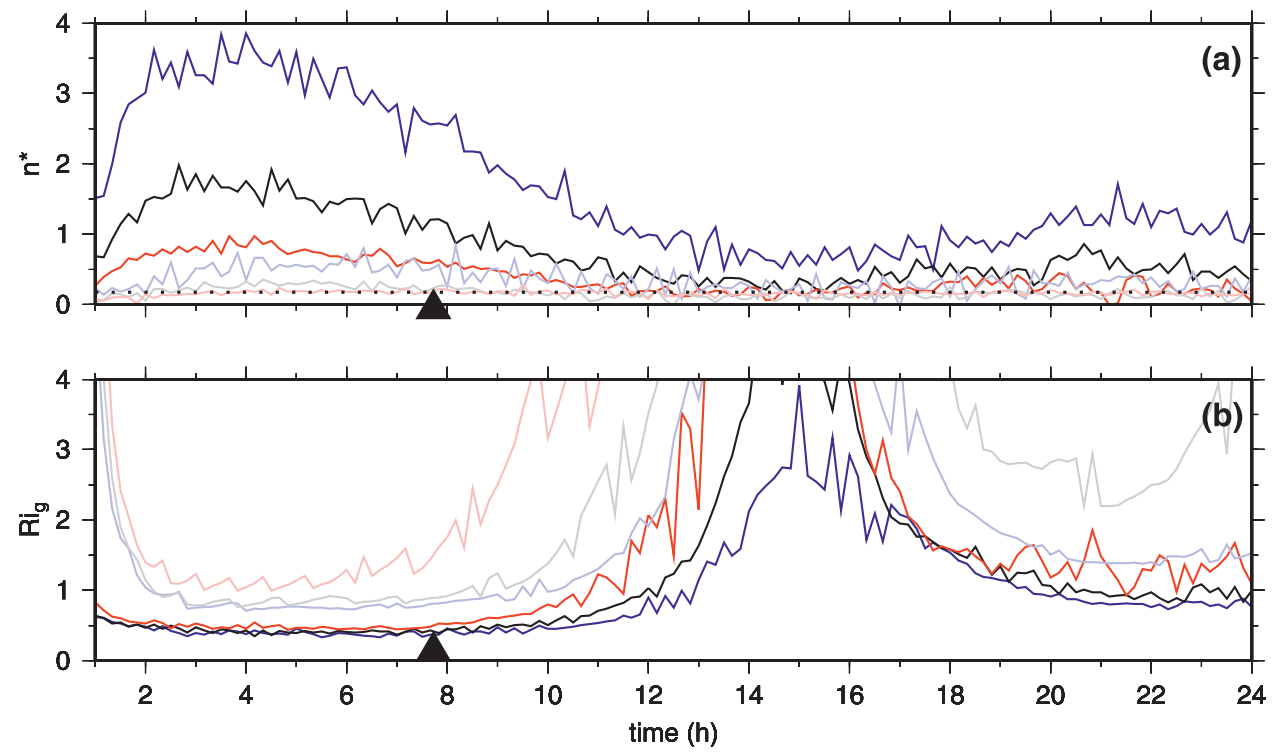

FIG. 4. Time series at the MLD $\left(\phi=55^{\circ} \mathrm{N}\right.$, without LC; light color: $u^{*}=0.01 \mathrm{~m} \mathrm{~s}^{-1}$; dark color: $u^{*}=$ $0.02 \mathrm{~m} \mathrm{~s}^{-1}$; blue: $Q_{0}=1.25 \times 10^{-7} \mathrm{~m}^{2} \mathrm{~s}^{-3}$; black: $Q_{0}=2.5 \times 10^{-7} \mathrm{~m}^{2} \mathrm{~s}^{-3}$; red: $Q_{0}=5 \times 10^{-7} \mathrm{~m}^{2} \mathrm{~s}^{-3}$ ). The time corresponding to $\pi / f$ is marked by an arrow, and the dotted horizontal line represents $n^{*}=0.17$ : (a) $n^{*}$ and (b) $\mathrm{Ri}_{g}$.

mixing within the mixed layer. When $\phi=0^{\circ}, S^{2}(z=-h)$ continues to increase until it reaches the oscillating stage $(t \sim 10 \mathrm{~h})$. Conversely, when $\phi=55^{\circ} \mathrm{N}, S^{2}(z=-h)$ initially increases until $t \sim \pi / 2 f$, then decreases thereafter, because the current velocity rotates from the direction of wind stress because of inertial oscillation. Although $S^{2}(z=-h)$ increases again at $t>\pi / f$, its magnitude is much smaller than the first peak because of the increased MLD.

To be consistent with this result (Figs. $1 \mathrm{~b}, \mathrm{c}$ ), $m_{0}$ in (1) should be 0 , contrary to the NK model, because $u_{*}$ does not vanish with time. It contrasts with the atmospheric boundary layer in which $m_{0}$ remains constant during the convective growth, regardless of latitude (Moeng and Sullivan 1994; Driedonks 1982; Noh et al. 2003).

Meanwhile, $m_{s}$ in (1) need not be 0 , because $\Delta \mathbf{U}$ goes to 0 too. An evaluation of $m_{s}$ in (1), with $n=0.17$ and $m_{0}=0$, shows that the last term properly parameterizes the effect of velocity shear at the MLD (Fig. 3a), although large fluctuations occur at $t \gg \pi / f$, as both $-\overline{b w}_{h}-n Q_{0}$ and $w_{e}(\Delta \mathbf{U})^{2} / h$ approach 0 . The value of $m_{s}$ is close to that suggested in the NK model $\left(m_{s} \cong 0.48\right)$.

It is expected that the disappearance of $S^{2}(z=-h)$ may affect the values $\mathrm{Ri}_{g}$ and $\mathrm{Ri}_{b}$ at $z=-h$. Figures $3 \mathrm{~b}-\mathrm{d}$ show the variation of $\mathrm{Ri}_{g}, \mathrm{Ri}_{b}$, and $\mathrm{Ri}_{b}^{*}$ at $z=-h$. Until $t \sim \pi / f$, both $\mathrm{Ri}_{g}$ and $\mathrm{Ri}_{b}$ remain constant. When $t>\pi / f$, however, they increase rapidly, eventually achieving very large values. Although they return to smaller values again after $t \sim 2 \pi / f$, the values are much larger than the original critical values. On the other hand, Ri* avoids a rapid increase at $t>\pi / f$, because the presence of the term $V_{t}^{2}$ suppresses the sharp increase of $\mathrm{Ri}_{b}^{*}$ even in the absence of $S^{2}(z=-h)$. Even if $\mathrm{Ri}_{b}^{*}$ is evaluated at the depth at which $\overline{b w}$ disappears below $h$, as it is usually done in the KPP model, it still remains invariant with time at a slightly larger value.

It is also found that the general feature, shown in Figs. $1-3$, remains largely invariant regardless of $u_{*}$ and $Q_{0}$ (Fig. 4). The values of $n^{*}$ remain larger than $n$ at $t>\pi / f$ for large $u_{*}$ and small $Q_{0}$, but this is attributed to the large magnitude of $w_{e}(\Delta \mathbf{U})^{2} / h$, rather than $u_{*}^{3} / h$, in (1).

\section{b. Effects of the criteria on the predictability of $M L D$}

In the real ocean, wind stress and surface heat flux undergo complex variation. Nonetheless, the present simulation results strongly suggest that the mean contribution from wind forcing becomes smaller at high-latitude ocean, because there the time scale of wind stress is usually much longer than $\pi / f$ (see, e.g., Davis et al. 1981a; Gill 1982; Qiu et al. 2004). It implies that the criterion (1) can cause an overestimation of MLD at high latitudes, whereas the criteria (2) and (3) can cause an underestimation.

Indeed the model results at the ocean station Papa $\left(\phi=50^{\circ} \mathrm{N}\right)$ reveals that, during winter, the MLD is seriously overestimated by the NK model and underestimated by the PWP model, as shown in Fig. 5 (Martin 1985; Kantha and Clayson 1994). The corresponding overestimation and underestimation of MLD from these models have been widely reported (Gaspar 1988; Large et al. 1994; D'Alessio et al. 1998; Qiu and Chen 2006). It 

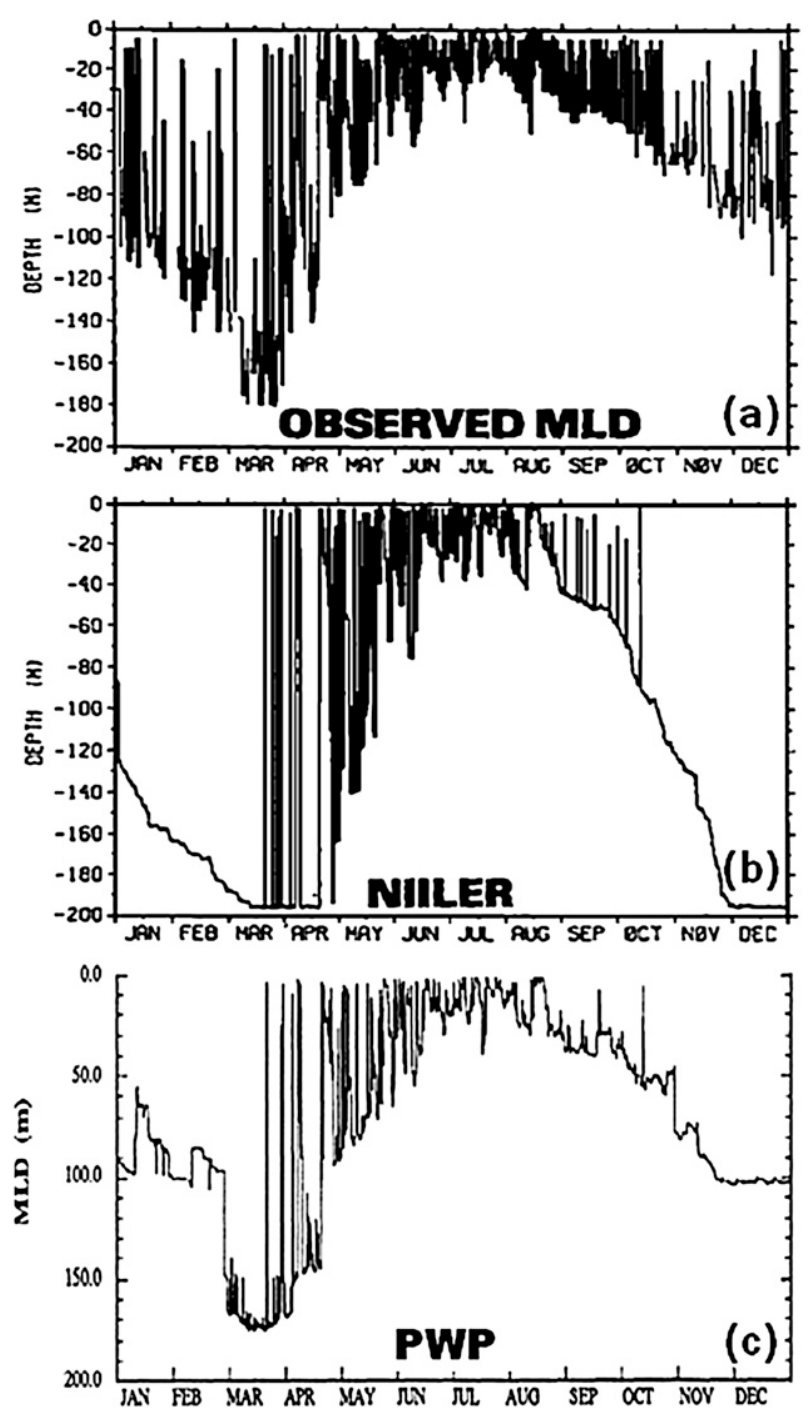

FIG. 5. Seasonal variation of MLD at station Papa for 1961: (a) observation, (b) NK model (from Martin 1985), and (c) PWP model (from Kantha and Clayson 1994).

is also important to notice that the NK model predicts MLD properly under storm conditions with resonant forcing but overestimates it under normal condition (Davis et al. 1981b), a result consistent with the present analysis.

Davis et al. (1981b) showed that $m_{0}$ in (1) is necessary in the NK model to avoid too shallow MLD and too high sea surface temperatures (SST) under the surface heating, when the wind stress is weak. It is consistent with the fact that neglecting the effects of wave breaking (WB) and LC leads to too shallow MLD and too high SST under the surface heating (Noh et al. 2009). Note that, if velocity shear is negligible within the mixed layer, as assumed in bulk models, the only contribution of wind stress to turbulence generation comes through WB and LC. It implies that the term with $m_{0}$ in (1) represents a contribution from these sources. However, many studies suggest that the effect of strong mixing by WB and LC may be limited to the near-surface layer (Craig and Banner 1994; Noh et al. 2004; Thorpe 2004). Accordingly, their contribution should decrease with depth, and the term $2 m_{0} u_{*}^{3} / h$ in (1) needs to be modified to $2 m_{0} u_{*}^{3} e^{z / \delta} / h$ with an appropriate depth scale $\delta$. The contribution from the velocity shear induced by wind forcing is represented by the term $m_{s} w_{e}(\Delta \mathbf{U})^{2} / h$.

\section{Conclusions}

In the present paper, the criteria for the deepening of the convective mixed layer in the widely used mixed layer models, such as the NK model, the PWP model, and the KPP model, are examined by analyzing LES data. It is found that the contribution from wind stress decreases with time as a result of inertial oscillation in the extratropical ocean, contrary to the NK model, and it leads to a rapid increase of the bulk and gradient Richardson number at the MLD well above the critical values used in the PWP model. Meanwhile, the critical Richardson number in the KPP model turns out to be relevant.

The present analysis clarifies the problem associated with too deep MLD predicted by the NK model and too shallow MLD predicted by the PWP model in winter, which has been widely reported (Martin 1985; Kantha and Clayson 1994; Gaspar 1988; Large et al. 1994; D'Alessio et al. 1998; Qiu and Chen 2006). A possible modification of the NK model is suggested.

Acknowledgments. This work was supported by the Korea Research Foundation Grant funded by the Korean Government (MOEHRD, Basic Research Promotion Fund; KRF-2007-C00788) and by the National Research Foundation of Korea Grant funded by the Korean Government (MEST; NRF-2009-C1AAA001-0093068). Supercomputing was supported by KISTI (KSC-2008S01-0009).

\section{REFERENCES}

Ayotte, K. W., and Coauthors, 1996: An evaluation of neutral and convective planetary boundary-layer parameterizations relative to large eddy simulations. Bound.-Layer Meteor., 79, 131-175.

Craig, P. D., and M. L. Banner, 1994: Modeling wave-enhanced turbulence in the ocean surface layer. J. Phys. Oceanogr., 24, 2546-2559.

Craik, A. D. D., and S. Leibovich, 1976: A rational model for Langmuir circulations. J. Fluid Mech., 73, 401-426.

Crawford, G. B., and W. G. Large, 1996: A numerical investigation of resonant inertial response of the ocean to wind forcing. J. Phys. Oceanogr., 26, 873-891.

D'Alessio, J. S. D., K. Abdella, and N. A. McFarlane, 1998: A new second-order turbulence closure scheme for modeling the ocean mixed layer. J. Phys. Oceanogr., 28, 1624-1641. 
Davis, R. E., R. de Szoeke, and P. P. Niiler, 1981a: Variability in the upper ocean during MILE. Part I: The heat and momentum balances. Deep-Sea Res., 28, 1427-1451.

$\longrightarrow,-$, and $-1981 \mathrm{~b}$ : Variability in the upper ocean during MILE. Part II: Modeling the mixed layer response. Deep-Sea Res., 28, 1453-1475.

Denbo, D. W., and E. D. Skyllingstad, 1996: An ocean large-eddy simulation model with application to deep convection in the Greenland Sea. J. Geophys. Res., 101, 1095-1110.

Doran, J. C., 2004: Characteristics of intermittent turbulent temperature fluxes in stable conditions. Bound.-Layer Meteor., 112, 241-255.

Driedonks, A. G. M., 1982: Models and observations of the growth of the atmospheric boundary layer. Bound.-Layer Meteor., 23, 283-306.

Gaspar, P., 1988: Modeling the seasonal cycle of the upper ocean. J. Phys. Oceanogr., 18, 161-180.

Gill, A. E., 1982: Atmosphere-Ocean Dynamics. Academic Press, $325 \mathrm{pp}$.

Kantha, L. H., and C. A. Clayson, 1994: An improved mixed layer model for geophysical applications. J. Geophys. Res., 99, 25 23525266.

Large, W. G., and G. B. Crawford, 1995: Observations and simulations of upper-ocean response to wind events during the Ocean Storms Experiments. J. Phys. Oceanogr., 25, 2831-2852.

— J. C. McWilliams, and S. C. Doney, 1994: Oceanic vertical mixing: A review and a model with a nonlocal boundary layer parameterization. Rev. Geophys., 32, 363-403.

Li, M., C. Garrette, and E. Skyllingstad, 2005: A regime diagram for classifying turbulent large eddies in the upper ocean. DeepSea Res. I, 52, 259-278.

Mahrt, L., 1999: Stratified atmospheric boundary layers. Bound.Layer Meteor., 90, 375-396.

Martin, P. J., 1985: Simulation of the mixed layer at OWS November and Papa with several models. J. Geophys. Res., 90, 903-916.

Moeng, C. H., and P. P. Sullivan, 1994: A comparison of shear- and buoyancy-driven planetary boundary layer flows. J. Atmos. Sci., 51, 999-1022.

Niiler, P. P., and E. B. Kraus, 1977: One-dimensional models of the upper ocean. Modelling and Prediction of the Upper Layers of the Ocean, E. B. Kraus, Ed., Pergamon, 143-172.
Noh, Y., and H. J. Kim, 1999: Simulations of temperature and turbulence structure of the oceanic boundary layer with the improved near-surface process. J. Geophys. Res., 104, 15 62115634.

, W. G. Cheon, S. Y. Hong, and S. Raasch, 2003: Improvement of the K-profile model for the planetary boundary layer based on large eddy simulation data. Bound.-Layer Meteor., 107, 401-427.

- H. S. Min, and S. Raasch, 2004: Large eddy simulation of the ocean mixed layer: The effects of wave breaking and Langmuir circulation. J. Phys. Oceanogr., 34, 720-735.

—, G. Goh, S. Raasch, and M. Gryschka, 2009: Formation of a diurnal thermocline in the ocean mixed layer simulated by LES. J. Phys. Oceanogr., 39, 1244-1257.

Price, J. F., R. A. Weller, and R. Pinkel, 1986: Diurnal cycles of current, temperature and turbulent diffusion in a model of the equatorial upper ocean. J. Geophys. Res., 91, 8411-8427.

Qiu, B., and S. Chen, 2006: Decadal variability in the formation of the North Pacific Subtropical Mode Water: Oceanic versus atmospheric control. J. Phys. Oceanogr., 36, 1365-1380.

, — - and P. Hacker, 2004: Synoptic-scale air-sea flux forcing in the western North Pacific: Observations and their impact on SST and the mixed layer. J. Phys. Oceanogr., 34, $2148-2159$.

Raasch, S., and D. Etling, 1991: Numerical simulation of rotating turbulent thermal convection. Contrib. Atmos. Phys., 3, $1-15$.

Skyllingstad, E. D., W. D. Smith, and G. B. Crawford, 2000: Resonant wind-driven mixing in the ocean boundary layer. J. Phys. Oceanogr., 30, 1866-1890.

Stull, R. B., 1986: An Introduction to Boundary Layer Meteorology. Kluwer Academic, 478 pp.

Sullivan, P. P., J. C. McWilliams, and W. K. Melville, 2007: Surface gravity wave effects in the oceanic boundary layer: Large-eddy simulation with vortex force and stochastic breakers. J. Fluid Mech., 593, 405-452.

Thorpe, S. A., 2004: Langmuir circulation. Annu. Rev. Fluid Mech., 36, 55-79.

Wang, D., 2003: Entrainment laws and a bulk mixed layer model of rotating convection derived from large-eddy simulations. Geophys. Res. Lett., 30, 1929, doi:10.1029/2003GL017869. 